\title{
Geschichte und Psychotherapie im Austausch: Lebensgeschichten mit Oral-History-Interviews erfassen
}

\author{
Albert Lichtblau
}

Online publiziert: 30 . September 2021

(C) Der/die Autor(en) 2021

Zusammenfassung Dieser Beitrag für die Zeitschrift für Psychodrama und Soziometrie reflektiert die Methode Oral History, die sich mit der Aufzeichnung, Archivierung und Auswertung von lebensgeschichtlichen Interviews befasst. Für die Aufarbeitung der Gewaltgeschichte von zwei Weltkriegen und fortdauernder Massengewalt im 20. Jahrhundert waren die Erfahrungen aus der therapeutischen Arbeit mit dadurch traumatisierten Menschen eine wichtige Stütze. Das Wissen über historische Rahmenbedingungen wiederum kann wichtige Hinweise für die therapeutische Arbeit geben, um die Darstellungsweisen individueller Geschichten besser zu verstehen.

Schlüsselwörter Oral History · Methodik · Kollektive Erinnerung · False memory · Schweigen · Trauma · Genozide · Generationen · Postmemory · Interdisziplinarität $\cdot$ Psychodrama $\cdot$ Holocaust $\cdot$ Heimkinder

Univ. Prof. em., Dr. Albert Lichtblau ( $ه)$

Paris Lodron University of Salzburg, Rudolfskai 42, 5020 Salzburg, Österreich

E-Mail: albert.lichtblau@sbg.ac.at 


\title{
History and psychotherapy in exchange: Recording difficult life stories with oral history interviews
}

\begin{abstract}
This contribution for the journal Zeitschrift für Psychodrama und Soziometrie reflects the method of oral history, which deals with the recording, archiving and analysis of life history interviews. Considering the history of violence from two world wars in the 20th century and ongoing mass violence, experiences from therapeutic practice with people traumatized by these violent events were an important support. On the other hand, knowledge of historical framework conditions in therapeutic work can certainly provide important information in order to better understand how individuals present their life stories.
\end{abstract}

Keywords Oral History · Methodology · Collective memory · False memory · Silence · Trauma · Genocide - Generation allocations · Postmemory · Interdisciplinarity $\cdot$ Shoah $\cdot$ Psychodrama $\cdot$ Institutionalized children

Oral History, die mittlerweile in den Geschichtswissenschaften eine übliche Methode ist, war nicht die erste, mit deren Hilfe Menschen befragt wurden, um ihre Aussagen und Handlungen wissenschaftlich zu analysieren. Anthropologie und Ethnografie spielten eine, wenn auch zum Teil kolonial bedingt problematische Vorreiterrolle. Die Oral-History-Methode der zuerst mit Tonbändern aufgenommenen mündlichen Befragung etablierte sich zunächst in den USA. In den 1970er und 1980er-Jahren wurde sie bereits global mit unterschiedlichen institutionellen Einbettungen praktiziert. Während beispielsweise in den USA oder in Kanada eigene Institute für Oral History entstanden, fehlen derartige, ausschließlich auf Oral History ausgerichtete Universitätsinstitute im deutschsprachigen Raum bislang. ${ }^{1}$ Der Vorteil der NichtInstitutionalisierung ist die Eingebundenheit in breitere methodische Zugänge und Quellennutzung. Die Nachteile sind dennoch eklatant, denn es fehlt an notwendiger Infrastruktur wie Archiven und vor allem an einer adäquat professionellen Ausbildung (Apel 2018). ${ }^{2}$ Mit diesen Mankos begann ich in den frühen 1980er-Jahren ohne universitäre, praxisorientierte Vorbildung mit anderen Kolleginnen und Kollegen Menschen über ihre Lebensgeschichte zu befragen. Das Interesse an Oral History entsprang dem Wunsch, sich aus der herrschaftsfixierten Geschichtsschreibung zu befreien und die Stimmen der Menschen zu hören, deren Geschichte bis dahin weitgehend ignoriert worden war. Neben Giving Voice war Dig Where you Stand ein weiteres Schlagwort. Das Motto „Grabe wo du stehst“ wurde vom schwedischen Autor Sven Lindqvist übernommen und beeinflusste die Geschichtsbewegungen im deutschsprachigen Raum ab den 1980er-Jahren. Schnell war klar, dass in Öster-

\footnotetext{
1 Eine Ausnahme ist das Institut für Geschichte und Biographie der FernUniversität Hagen, von dem auch die für diesen Forschungsbereich wichtigste deutschsprachige „Zeitschrift für Biographieforschung, Oral History und Lebensverlaufsanalysen - BIOS“ herausgegeben wird.

2 In Österreich spielt die Österreichische Mediathek eine wichtige Rolle für die Archivierung von Oral History-Interviews: https://www.mediathek.at, 07.12.2020.
} 
reich oder Deutschland beim Graben in der Vergangenheit das „Braune“ der NSZeit hochkam und damit die auf uns allen lastende Gewaltvergangenheit.

Der persönliche Kontakt mit Personen, die ihre Version der Geschichte erzählten, zwang automatisch dazu, uns gleichsam mit Fragen von Generation und generationsübergreifenden Blicken auf die Vergangenheit zu beschäftigen. Der zu dieser Zeit schwelende Generationskonflikt betraf auch die Interpretation der NS-Vergangenheit, das Gemeinsame und Trennende blieben Wegbegleiter der Forschungslandschaft. Die in der Geschichtswissenschaft wichtig gewordenen Begriffe Generation oder kollektiv (wie im Falle kollektiven Gedächtnisses oder kollektiver Identität) wurden und werden allerdings oft fahrlässig generalisierend strapaziert. Denn ein gemeinsam, also kollektiv erlebtes Ereignis ist oft die Ursache für den getrennten Erfahrungshorizont: Schon bei den Lebensgeschichten von Kriegskindern des Zweiten Weltkriegs gibt es große Unterschiede, etwa ob die Väter aus dem Krieg zurückgekommen sind oder nicht, in welcher Funktion Eltern am Kriegsgeschehen oder den Kriegsverbrechen teilgenommen haben, wie fern oder nah sie den Parteiorganisationen der NSDAP gestanden bzw. ins Visier der Verfolgung geraten sind. Die klassische, eindeutige Trennlinie Opfer - TäterIn - Zusehende (Bystander) wird inzwischen hinterfragt, da sie den dynamischen Prozess eines Sinneswandels oder den kaum erklärbaren mehrfachen Rollenwechsel (etwa Rollenwechsel vom Bystander zu TäterIn zu Bystander-Hilfestellung) nicht berücksichtigt (Bajohr und Löw 2016). Das macht alles noch komplizierter, denn der Wunsch nach einfachen Zuschreibungen ist stark ausgeprägt.

Wir können durchaus von der Kriegsgeneration sprechen, den Kriegskindern oder den Nachkriegskindern, die sich meist schon mit den 68ern überschneiden, der Generation X (die Generation nach dem Babyboom Ende der 1960er bis Anfang der 1980er), die darauffolgende Generation $Y$ (oder Millennials) usw. Damit sind Klischeevorstellungen verbunden, die nur schwer zu konterkarieren sind. Blicken wir etwa auf die 68er: Nicht die Beatles oder die Rolling Stones dominierten damals den Musikmarkt, sondern die meistverkaufte Single in der BRD war tatsächlich das Lied „Mama“ (mit dem Liedtext ,... nie vergessen/Was ich an dir hab besessen/Dass es auf Erden nur Eine gibt/Die mich so heiß hat geliebt/Mama ..."). Bei den jeweils generationsprägenden Ereignissen kommen noch weitere Faktoren hinzu wie jene des Alters, der sozialen Schichtzugehörigkeit, der politischen, religiösen, sexuellen Orientierung oder des Geschlechts, die wiederum die Fragen an die Vergangenheit und die Familiengeschichten prägten.

Zurück zur konkreten Arbeit der Oral History: Schon bei meinen frühen Interviews für das Projekt Schmelztiegel Wien Mitte der 1980er-Jahre (vgl. John und Lichtblau 1990) hätte mir klar sein müssen, dass ich (ebenso wie alle anderen InterviewerInnen) jederzeit jemandem begegnen konnte, der oder die grausame Gewalt erfahren oder ausgeübt hatte. Meine diesbezüglich schlimmste Erfahrung war ein Interview, bei dem ich damals kurzfristig als Interviewer einsprang und nicht wusste, dass der Interviewte aus der Gruppe der tschechischen Volksgruppe im KZ interniert und dort gefoltert worden war. Hätte ich es gewusst, wären meine Fragen ganz andere gewesen. So aber beschrieb der Mann zum ersten Mal seine Foltererfahrung im Interview, noch dazu sehr bildhaft und schien emotional völlig aufgelöst. Das war keine gute Konstellation für einen noch unerfahrenen und nicht darauf 
vorbereiteten Interviewer. Meine Oral-History-Reflexionen kreisen seither immer wieder um diese Situation der völligen Überforderung im Frühstadium meiner Arbeit. Bei dem beschriebenen Interview konnte ich nur intuitiv reagieren: Ich sagte dem Mann, er müsse nicht weiter erzählen, doch der Interviewte war nicht mehr zu stoppen. Ich versuchte, selbst nicht mit in den Schmerz hineinzugehen, sondern in der Rolle des Verantwortung Übernehmenden das Ende der Erzählung beobachtend abzuwarten und danach das Interview auslaufen zu lassen. Der Interviewte sollte sich beruhigen können, weswegen ich nach dem Ende der KZ-Beschreibung und dem eher abrupten Ende des Interviews mit dem Interviewten über seinen gegenwärtigen Alltag sprach, um ihn in der Gegenwart zurücklassen zu können. Seither war mir völlig klar, dass bei lebensgeschichtlich orientierten Interviews jederzeit traumatische Erfahrungen aufbrechen können. Eine Konsequenz daraus war es für mich, nie Termine unmittelbar nach dem Interview zu vereinbaren, um notfalls über ausreichend Zeit für Hilfe verfügen zu können (Vickers 2019).

Zum Glück waren in meinem Umfeld von Beginn an zahlreiche Bekannte wie Hildegard Pruckner (vgl. auch den Beitrag „Psychodrama mit traumatisierten Menschen" von Pruckner in diesem Heft: Pruckner 2021) oder die in New York lebende Psychologin Dorit B. Whiteman (Whiteman 1995) mit therapeutischer Kompetenz, die ich immer wieder zu Rate ziehen konnte, etwa zur Frage wie sie mit schwierigen, traumabezogenen Gesprächssituationen umgehen. Es war mir von Anbeginn an klar, dass wir als Oral Historians nicht nur Verantwortung für die Menschen, die wir befragen, übernehmen, sondern auch für uns als Fragende, da die Gefahr einer sekundären Traumatisierung bei einer intensiven Arbeit mit den von Gewalterfahrung geprägten Erinnerungen gegeben ist. Das betraf vor allem jene Interviewenden, die ausschließlich Opfer der NS-Verfolgung oder Menschen mit Kriegs- oder Fluchterfahrungen befragten. Unverständlich war mir die innerhalb der Oral-History-Community obligatorische Vorgabe des empathischen Zuhörens, ohne zugleich darüber zu reflektieren, was das mit „uns“ als Zuhörenden macht. Doch das betraf nicht nur die Oral History, sondern die Geschichtswissenschaft insgesamt. Um ein Beispiel aus einem anderen Bereich zu geben: Die von der Regierung eingesetzte Historikerkommission der Republik Österreich (1998-2003) beauftragte zahlreiche, auch viele junge Forschende, sich mit dem Vermögensentzug in der NS-Zeit und den Folgewirkungen zu befassen. An eine begleitende Supervision wurde jedoch nicht gedacht, was ich für völlig unverantwortlich hielt. Wer jeden Tag die eiskalten Akten von Demütigung, sozialer Marginalisierung, Entmenschlichung der Opfergruppen durch Beraubung, Vertreibung bis hin zu Mord durcharbeitet, kann an der Menschheit zu zweifeln beginnen, also ,verzweifeln“.

Da ich ab den 1990er-Jahren begann, an der Universität Oral History zu unterrichten, versuchte ich für die Ausbildung einen verantwortungsvollen Umgang zu finden und diskutierte meine Herangehensweise immer wieder auf Konferenzen (Lichtblau 2018). Der Erfahrungsaustausch war sehr wichtig, da Kollegen und Kolleginnen über Gespräche mit Menschen berichteten, die zahlreiche andere Gewalterfahrungen erfahren hatten (Genozide an der armenischen Bevölkerung, in Ruanda, Massengewalt in Kambodscha oder Kriege im ehemaligen Jugoslawien, afro-amerikanische Gewalterfahrungen auf Grund von Rassismus). Wie wir uns vor Überforderung schützen können, wurde für mich ein wichtiges Thema, weswegen 
ich einen Artikel als Gegenposition zum Empathie-Pathos „Keeping Distance“ nannte (Lichtblau 2002) und andere Oral Historians dazu einlud, schwierige Situationen bei Interviews zu beschreiben, um jenen mit wenig Berufspraxis Handlungsmöglichkeiten des Umgangs damit zu vermitteln. Eine Kollegin beschrieb etwa, wie sie für das Mauthausen-Projekt in den USA einen Überlebenden interviewen wollte, dessen Ehefrau ebenfalls eine KZ-Überlebende war. Nach freundlichen Vorgesprächen wurde sie an einem vereinbarten Ort von den beiden abgeholt, danach eskalierte die Situation im Auto. Die Ehefrau wurde misstrauisch, verlangte einen Ausweis, vertraute der Interviewerin nicht. Als die Kollegin unterwegs endlich aussteigen konnte, stieg auch die Ehefrau aus: ,... she left the car and started running down the street, pulling her hair and screaming repeatedly and pointing at me, someone call the police,- - that woman, that woman. 'I was walking as quickly as I could, shaken, not knowing which way to go, in a neighborhood where I felt unsafe. Passers-by looked at me and neighbors peered from their windows.“ (Ghitis 2004) Später kam der Mann, der eigentlich interviewt hätte werden sollen, zurück und entschuldigte sich für das Verhalten seiner Partnerin. Obwohl die Interviewerin bereits viel Erfahrung in dem Bereich hatte, konnte sie die Geschehnisse nicht verhindern. Später suchte sie nach Hinweisen aus der Traumaforschung, um das Verhalten der Ehefrau zu verstehen, wie z.B.: ,After a traumatic experience, the human system of selfpreservation seems to go onto permanent alert, as if the danger might return at any moment.“ (ebd.) Das Interview hätte 2002, also ein Jahr nach 9/11 stattfinden sollen; die terroristische Gefahr schien noch allgegenwärtig. Die geschilderte Situation war eine große Überforderung für die Interviewerin. An dem Abend half ihr telefonisch das ,informal support team“ mehrerer KollegInnen, die ebenfalls für das Mauthausen-Projekt in den USA interviewten.

Derart extrem angespannte Situationen passieren zwar kaum, aber sie sind möglich. Umso wichtiger ist es mir, diese Worst-Case-Szenarien in Oral-History-bezogenen Lehrveranstaltungen anzusprechen und Handlungsmöglichkeiten anzubieten. Das betrifft auch die Signale der Körpersprache. Inzwischen wird provokant gefragt: „How Dangerous is Oral History“ und das gilt nicht nur für die Befragten, sondern eben auch für die Fragenden (Rickard 1998). Auf Grund langjähriger Beobachtung habe ich den Eindruck, dass Personen mit ausgeprägten Traumafolgewirkungen ein Oral-History-Interview eher ablehnen. Dass sie sich der Gefahr der Selbstbeschädigung bewusst sind, könnte einer der Gründe dafür sein. In Übungsinterviews von Studierenden meiner Lehrveranstaltungen ist es seit den 1990er-Jahren nur einmal vorgekommen, dass ein Interview abgebrochen werden musste, nämlich als es offensichtlich wurde, dass die Interviewte Opfer einer Vergewaltigung war und sie anscheinend nicht damit gerechnet hatte, dass die Erinnerung daran so massiv reaktiviert hätte werden können. Insofern ist es für uns Oral Historians auch wichtig, dass wir uns mit dem Begriff „Trauma“ abseits einer modisch-inflationären Verwendung befassen und wissen, dass es in Gesprächen und Situationen Trigger wie (Erinnerungen an) Gerüche, Töne oder Objekte ${ }^{3}$ geben kann, die zu Retraumatisierung oder zu heftigen Abwehrreaktionen führen können.

\footnotetext{
3 Z.B. der Geruch nach verbranntem Fleisch, Sirenengeheul oder Böllerschüsse, Stiefel, Hunde etc.
} 
Für jene, die sich als Oral Historians mit Opfern von Genoziden oder Massengewalt befassten, war ein interdisziplinärer Dialog mit Kolleginnen und Kollegen aus den Bereichen Psychologie und Therapie essentiell und sehr hilfreich (von Plato 2004). Inspirierend waren für mich Konferenzen der ISPP (International Society for Political Psychology), da dort schon früh vergleichende Genozidforschung diskutiert und nicht nur die Rolle von Opfern, sondern auch von Tätern und Täterinnen analysiert wurde (Robins und Post 1997; Staub 1989, 2015). Bezogen auf die Geschichte der NS-Zeit blieb in der Oral History genau dieser Aspekt eine Schwachstelle, da TäterInnen uns de facto keine Interviews gaben und über ihre kriminellen Handlungen nicht sprachen. Deswegen verharmlosten die Gespräche mit Angehörigen der Kriegsgeneration (AnhängerInnen und MitläuferInnen des NS-Regimes, Passive) die über den Krieg hinausgehenden Gewaltakte weitgehend. Falls überhaupt von einer aktiven Teilnahme innerhalb der NSDAP und ihrer Untergliederungen gesprochen wurde, gab es Legitimationsstrategien vom Runterpegeln der eigenen Rolle, als wäre jemand selbst zum Opfer der Geschichte geworden, bis hin zur Verweigerung und zum Gesprächsabbruch mit einem „Das verstehst du nicht“, was in einem gewissen Sinn sogar stimmte. Je weniger die Erzählungen auf dem Prüfstand historischer Faktizität standen, umso beliebiger konnten Befragte ihre Erinnerungen gestalten. Insofern war es nicht überraschend, dass die 2002 veröffentlichte Studie „Opa war kein Nazi“" dies empirisch belegte: $26 \%$ der Befragten vermuteten über ihre in der NS-Zeit aktiv handelnden Eltern oder Großeltern, sie hätten Verfolgten geholfen, $13 \%$ meinten, diese seien im Widerstand aktiv gewesen und nur 3\% der Eltern und Großeltern hätten antijüdische Einstellungen gehabt, auf der anderen Seite hätten $65 \%$ der Angehörigen ,viel im Krieg erlitten“ (Welzer 2009, S. 75-77). Wir Oral Historians haben uns mit den zahlreichen narrativen Mustern beschäftigt, etwa wenn Kriegsteilnehmende auf das Format der standardmäßig oftmals erprobten Anekdote zurückgriffen, um überhaupt etwas von ihren Erlebnissen erzählen zu können.

Diese Beobachtung konnte ich auch in der Lehre machen, wenn Studierende Gespräche innerhalb der Familien führten. In den erzählten Erinnerungen beschrieben sich Befragte in einer Form, wie es wünschenswert gewesen wäre: als offen, hilfsbereit, tolerant, wenn nicht gar widerständig - so als hätte sich der demokratische Lernprozess erfolgreich in die Umgestaltung der Lebenserinnerungen eingeschrieben. Ob wir das als Verleugnen, Verschonen, Unwahrheit, Vergessen, Verdrehen, gelernt, als Ausreden oder einfach nur Verschweigen klassifizieren, ist im Einzelfall schwer zu beurteilen. Nur wenn historische Dokumente, seien es Briefe, Lebensläufe, Einvernahmen oder Aussagen anderer vorliegen, können wir dies genauer bewerten (Reiter 2006). Oft tauchen derartige Dokumente erst nach dem Ableben auf, und mitunter scheint der Spruch von der „Leiche im Keller“ plötzlich sehr real. Das Nichtwissen über Vorfahren führte aber auch zu Unsicherheiten und Ängsten, sich manchmal verhärtenden Vermutungen. Ein Muster war es, die in der NS-Zeit aktiven Mütter, anders als die Väter, im Familiengedächtnis mit einer Unschuldsvermutung eher auf die Seite der Opfer zu rücken (Reiter 2010, S. 72-75).

Es bleibt ein Phänomen, dass das Schweigen, wenn auch aus völlig anderen Gründen, nicht nur bei Täterinnen und Tätern oder Bystander-Familien auftrat, sondern selbst in Familien der NS-Opfer. Einen Durchbruch brachte der Memory-Comic „Maus“ von Art Spiegelman in den 1980er-Jahren. Als Kind zweier KZ-Überleben- 
der - seine Mutter beging 1968 Suizid - beschrieb und zeichnete Spiegelman die von ihm aufgenommenen Gespräche mit seinem Vater und zeigte drastisch, wie sehr die Gewalterfahrungen der Eltern fortwirkten und sich auf ihn übertrugen (Spiegelman 2011). Dass er Juden als Mäuse, Deutsche als Katzen, Polen als Schweine etc. darstellte, löste heftige Debatten über die Darstellbarkeit aus. Spiegelman war in dieser Zeit eine Ausnahme und deswegen so wichtig: In vielen Opferinterviews bekamen wir zu hören, dass die Personen das erste Mal mit jemandem über ihre Erfahrungen in der NS-Zeit sprachen, meist weil sie ihre Kinder und Familien damit nicht belasten wollten. Das Erzählen fiel den Betroffenen oft nicht leicht und kostete Überwindung. Eine Motivation dafür war in höherem Alter der Erinnerungsdruck, nämlich dass die Überlebenden angesichts der ums Leben gekommenen Opfer eine Pflicht hätten, sich zu erinnern, damit so etwas Furchtbares nicht wieder passieren könne. ${ }^{4}$ Ein Beispiel aus der Praxis: Als es gelang, 1993 aus Salzburg stammende jüdische Überlebende nach Salzburg einzuladen, hatten wir jeweils nur kurz Zeit für Interviews mit den Rückkehrenden, weswegen in einem Fall ausnahmsweise auch zwei Töchter im Hintergrund zuhörten. Wir führten das Interview zu zweit durch und ein Überlebender erzählte uns von seiner Internierung im KZ Dachau nach dem Novemberpogrom 1938. Seine beiden Töchter hörten schluchzend zu. Eigentlich bemühen wir uns, dass keine Familienmitglieder bei den Interviews anwesend sind, aber in diesem Fall war es nicht anders möglich. Ich bedaure es, dass der Kontakt damals abbrach: Was bedeutete dieses Gespräch für die Töchter (Berkowitz 1998)? Wie auch immer, wir konnten als Interviewende kein Ersatz für die eigenen Fragen an die Eltern oder Großeltern sein, auch wenn wir manchmal eine Frage gleichsam auftragsmäßig mit auf den Weg bekamen. Der israelische Psychologe Dan Bar-On und andere haben für das gegenseitige Schweigen in Familien die Formulierung der „doppelten Mauer“ verwendet, die schwer zu überwinden war, da selbst wenn eine Seite bereit gewesen wäre zu sprechen oder zuzuhören, es die andere Seite zu dieser Zeit nicht notwendigerweise auch war (Bar-On 1996, S. 21). Bar-On war interdisziplinär gut vernetzt und konnte viele Erkenntnisse in seine Arbeit miteinbeziehen, die auch uns als Oral Historians weiterhalfen. Einen wichtigen Hinweis übernahm er etwa von Dina Wardi, die in ihrer Arbeit über Therapie mit Kindern von Holocaust-Überlebenden beschrieb, dass Überlebende oft nur ein Kind für die Rolle der „Erinnerungskerze“ auswählten, und dieses damit überforderten. Ähnliches konnten wir bei anderen Interviews immer wieder beobachten, nämlich dass eine Person die Verantwortung für das Familiengedächtnis übernimmt (Wardi 1992).

Bei den Video-Interviews der für die von Steven Spielberg 1994 initiierten Shoah Visual History Foundation ${ }^{5}$ gab es am Ende immer eine Frage danach, was die Überlebenden der eigenen Familie und zukünftigen Generationen mit auf den Weg geben wollten. Danach wurden Mitglieder der Familie eingeladen hinzuzukommen und etwas zur interviewten Person zu sagen. Damit sollte gezeigt werden, dass trotz all

\footnotetext{
${ }^{4}$ Dass es dennoch - wie in Srebrenica oder Ruanda - dazu gekommen war, zermürbte all jene, die auf ein „Nie Wieder“ gehofft hatten.

5 https://sfi.usc.edu, 24.11.2020. Vgl. auch USC Shoah Foundation, Visual History Archive an der Freien Universität Berlin, https://www.vha.fu-berlin.de/archiv/sf/index.html, 24.11.2020, mit Transkripten aller deutschsprachig geführten Interviews.
} 
der Gewalt neue Familien entstanden waren und das Leben weiterging. Für uns als Interviewende waren dies Momente, in denen die unterschiedlich getragene Last der über Generationen hinausreichenden Lebensgeschichten besonders spürbar wurde. Um ein Beispiel zum Aspekt Nähe/Distanz zu geben, beziehe ich mich auf ein Interview meiner Kollegin Elisabeth Pozzi-Thanner mit einer jüdischen Überlebenden im Raum New York. Diese hatte als Kind in einer Kohlenkiste versteckt überlebt: „Nach außen hin hatte sie alles: ein schönes Haus, einen sorgenden Mann, gelungene erwachsene Kinder. Beim abschließenden Foto kamen sie dann alle dazu - aber sie saßen mit deutlichem Abstand zur Mutter. Davon hatte sie auch im Interview gesprochen - dass sie physische Nähe so schwer ertragen kann, und sich doch so sehr danach sehnt." "Um dies zu verstehen, waren die seit den 1970er-Jahren publizierten Arbeiten über die zweite und dritte Generation, etwa jene von Helen Epstein, eine wichtige Grundlage (Epstein 1979; Rosenthal 1997). Später kamen wichtige andere Forschungen hinzu, etwa von Marianne Hirsch, die den Begriff postmemory etablierte und familienbezogene Erinnerungen auch auf objekt- und mediengenerierte Impulse zurückführte. Relikte aus der Vergangenheit, seien es Fotografien, Objekte, aber auch Orte wie KZs werden neben den Erzählungen und im Falle des Ablebens von ZeitzeugInnen, den in Erinnerung gebliebenen Erzählungen, wichtige Anhaltspunkt im generationsüberschreitenden Transformationsprozess. Sie helfen dabei, mit den eigentlich unvorstellbaren Ereignissen verbunden zu bleiben (Hirsch 2012).

Auf Seite der Oral History war es immer klar, dass wir nicht der Versuchung erliegen dürfen, therapeutische Interventionen setzen $\mathrm{zu}$ wollen, denn das ist nicht unsere Kompetenz und Aufgabe. Zeitlich limitierte Gespräche können keine gezielte und kontinuierliche therapeutische Arbeit ersetzen, auch wenn einige Oral Historians damit kokettierten (McCarthy 2010). Wie erwähnt, habe ich enorm viel von den Gesprächen mit psychotherapeutisch Geschulten, dem Lesen ihrer Forschungsberichte, ihren Konferenzbeiträgen und Vorträgen für die Methode der Oral History mitnehmen können. Ein Beispiel von vielen war ein 2004 an der Universität Salzburg gehaltener Vortrag des israelischen Psychodramatikers Natan P. F. Kellermann über Traumabewältigung des Holocausts. In meiner Erinnerung hatte der Vortrag etwas Beklemmendes, als wäre der Vortragsort - Österreich - für Kellermann ein zusätzlich belastendes Element gewesen. Er ist ein Beispiel dafür, wie sehr die HolocaustÜberlebenserfahrung seiner aus Österreich stammenden Mutter in seinem Leben, aber auch in dem seiner Tochter präsent blieben. Er schrieb über sich: „Even though I was born and grew up in a country far removed from the horrors of the Holocaust, I have lived with its images for my entire life. My mother, Lilly (Frischmann) Kellermann, was a fully functioning and loving mother, and told me very little about her traumatic experiences. But her Holocaust trauma has painfully permeated my inner life. Imagining the Holocaust comes almost automatically to me. It's as if I have been there and have seen that before. Gruesome Holocaust associations fill my waking, and sleeping life and human suffering is a constant companion." (Kellermann 2009).

Was Kellermann für sich beschrieb - „as if I have been there“ - habe ich in meiner Arbeit mehrfach beobachtet. Bei einer Videodokumentation über Personen,

\footnotetext{
${ }^{6}$ E-Mail von Elisabeth Pozzi-Thanner, 28.10.2020.
} 
die für das Mauthausen Survivors Documentation Project interviewt worden waren und zur Eröffnung des Besucherzentrums am 11. Mai 2003 nach Mauthausen eingeladen wurden, begleiteten wir den 1926 in Krakau geborenen, nahe New York wohnenden Überlebenden Solomon J. Salat und seine beiden Töchter beim Besuch der Gedenkstätte. Wenn ich zuvor von einer ,doppelten Mauer“ des Schweigens geschrieben habe, so ist diese Familie ein Gegenbeispiel, denn Solomon J. Salat sprach mit den Töchtern von klein auf über seine Erfahrungen. Hier ein Ausschnitt aus dem Gespräch mit der älteren der beiden Töchter auf der Todesstiege der Gedenkstätte Mauthausen:

„Miriam Salat: ... my father was always telling me from the time I was a little girl. So I-just remember all the stories from-Actually I was very obsessed with the Holocaust when I was growing up, almost in an effort to relive it.

Albert Lichtblau: In what way?

Miriam Salat: ... to share the experience with him somehow.

Albert Lichtblau: You said to relive it. What do you mean?

Miriam Salat: I used try to imagine myself in the same situation. Or-I guess I had a certain amount of guilt as why I didn't grow up in this time and why he did. I was afraid that something is happening to me-like being raped-You know it's a little hyper vigilant. But-you know it went away. But certainly during the time being a teenager-13-14-15. I mean even now listen to his stories for me is a way of-I don't know-re-experience thing with him somehow ..." (Lichtblau 2006)

Ihre jüngere Schwester wiederum erzählte es ganz anders. Sie sei als Kind und Jugendliche der Geschichte des Vaters weitaus distanzierter gegenüber gestanden. Erst als sie selbst Mutter wurde, holten sie die Ängste ein: ,,You know it's not about me anymore. It's about - what if this happens again? You know what if people turn on the Jews again? You know - or all the people that died here. And what if my child's one of those people?" ${ }^{\text {"7 }}$ In Momenten wie diesen geht es nicht mehr um historische Wahrheit, sondern darum, wie sich die Erfahrungen auf die Nachkommen auswirken. Auch das ist eine essentielle Wahrheit. Für mich ist dieser, abseits von historischer Faktizität gelegene Aspekt sehr wichtig, denn erst so können wir besser verstehen, wie Menschen über ihr Leben erzählen: Das Vergangene hat immer eine Bedeutung für die Gegenwart, für die Zukunftserwartung und eben auch für die aktuelle Beschreibung des eigenen Lebens.

Es ist eine Binsenweisheit, dass Menschen ein miteinander erlebtes Ereignis unterschiedlich beschreiben, unterschiedliche Versatzstücke in Erinnerung behalten und damit das Ereignis unterschiedlich bewerten. Relevant wird dieses Phänomen bei jedem Gerichtsprozess, in dem Indizien zu einem Urteil führen sollen. Als Oral Historians haben wir selten die Möglichkeit, uns mit prozessähnlichen Settings wie einer Aussage unter Eid auseinanderzusetzen. Die Aufarbeitung von Übergriffen und Missbrauch wie jene an Schulen oder Erziehungsanstalten führten dazu, dass Personen aus den Bereichen Geschichte, Psychologie und Rechtswissenschaft zusammenarbeiteten. Mein Kollege Michael John gehört zu jenen, die sich

\footnotetext{
7 ebd.
} 
früh mit den Abgründen der Geschichte von Erziehungsanstalten befassten. In der Frühphase seiner Arbeit interviewte ich ihn einmal zur Frage, wann wir bei unserer Arbeit als Oral Historians an Grenzen stoßen. Er erzählte von Interviews mit ehemaligen Zöglingen und deren Beschreibung körperlicher und sexueller Gewalt. Das könne er so nicht publizieren, da das Personal der Heime bekannt sei, er mit Anzeigen rechnen müsse und den ehemaligen Zöglingen auf Grund ihrer Biografien ohnedies kein Glauben geschenkt werden würde. Erst das wiederholte Bekanntwerden der skandalösen Übergriffe und das darauf folgende Einsetzen von Untersuchungskommissionen machten es möglich, den Betroffenen Gehör zu verschaffen. In der 2013 erschienenen Arbeit für die Kommission Wilhelminenberg schrieb John über das vorangegangene Schweigen: „Auf diese Tatsache angesprochen, meinte ein ehemaliges Heimkind drastisch: ,Nein, ich habe mich nicht getraut. Es hat keiner gewusst, nicht einmal in der Familie hat jemand gewusst, was mir passiert ist. Wir sind schon so mundtot gemacht worden. Außerdem, es hätte eh keiner geglaubt. Wenn du das jemanden erzählst, du bist als Lügner dagestanden."“ (Helige et al. 2013, S. 250).

Für mehrere derartige Projekte wurden sowohl ehemalige Zöglinge bzw. Schüler und Schülerinnen als auch die Aufsichtspersonen befragt. Was nicht verwundern kann, so John, es war ,eine Haupttrennungslinie zu erkennen, ehemalige Heimkinder erinnerten sich anders als das Personal ... Es sind also Unschärfen oder offenkundige Fehlerinnerungen einerseits und divergente Darstellungen andererseits, die ein Problem bei der Auswertung der Interviews darstellten." (Helige et al. 2013, S. 222) In einem aufwendigen Verfahren stellte John die Transkripte in einer Parallelcollage nebeneinander, um so die unterschiedlichen Erzählweisen für die Analyse vergleichen zu können. Auf beiden Seiten kam es immer wieder vor, dass die Erinnerungen nicht mit den Details aus Akten übereinstimmten - sachliche Irrtümer, falsche Datierungen, Orts- oder Namensverwechslungen gehören generell zu Schwachstellen von Erinnerungen.

Für die konkrete geschichtswissenschaftliche Arbeit bedeutet dies, quellenkritisch vorzugehen, das „Aufgenommene“ mit anderen Aussagen und Dokumenten in Hinblick auf Validität zu vergleichen (Diekmann \& Schoeps 2002), bei Unklarheiten in Follow-up-Interviews nachzufragen. Es bedeutet aber auch, zwischen ,den Zeilen“ zu lesen, den Blick auf Vergangenes durch die Linse der Gegenwart zu verstehen und zu akzeptieren, dass es sich oft um aktuelle Verarbeitungsversionen der Vergangenheit handelt. Die Methode des Close Readings hilft, verschiedene Facetten der Ausdrucksformen und Nuancen zu erkennen. Dabei spielt der Einfluss der Interviewenden für die Gesprächsgestaltung eine gewichtige Rolle, das fiel mir besonders dann auf, wenn nicht selbstgeführte Interviews sekundäranalytisch herangezogen wurden (Halbmayr 2015). Bei der Formulierung von Fragen sind es oft einzelne Wörter oder kurze Phrasen, die den Antwortraum einschränken oder öffnen. In den Interviews verwenden Fragende oft folgende Phrase: „Können Sie mir ein wenig erzählen ...“ Damit signalisieren die Fragenden, dass es ihnen eventuell peinlich ist, die Frage überhaupt zu stellen und zugleich, dass sie keine ausführliche Antwort erwarten. Bei der Analyse der Interviews von anderen drängen sich sofort Fragen gegenüber den Fragenden auf: Warum wurde ein Thema nicht vertieft, 
ein Hinweis in der Antwort nicht für nachfolgende Fragen wahrgenommen usw. (Lichtblau 2022)?

Die Projekte über Heimkinder und Missbrauch zeigen, wie wichtig fächerübergreifende Kooperationen sein können. Deswegen sei abschließend ein gegenseitiges Lernen empfohlen, auch wenn ich mir der Grenzen durchaus bewusst bin - Gespräche und ein Einlesen können keine Ausbildung und langjährige Praxiserfahrung ersetzen. Die Kenntnis historischer Rahmenbedingungen kann in der therapeutischen Praxis dabei helfen, die Erfahrungshorizonte von Menschen aus anderen Zeiten und Regionen besser zu verstehen. Strukturgeschichtliche Kenntnisse geben sowohl Therapierenden als auch Therapierten Hinweise zur Einordnung spezifischer Muster von Erfahrungen und Verarbeitungsformen. An dieser Stelle sei auf den Wert der Arbeit mit Oral-History-Interviews und Lebensgeschichten hingewiesen: Lebenserinnerungen können Menschen in Therapie zeigen, dass sie nicht alleine sind mit ihren Erfahrungen, auch wenn sie sich oft so fühlen. Im Psychodrama wird dies Sharing genannt. Es stellt eine wesentliche und bedeutsame Technik des Psychodrama-Verfahrens in Gruppen- und Einzeltherapie dar. Besonders in der Gruppentherapie entfaltet das Sharing häufig heilsamen Charakter. Aber auch in der Einzeltherapie wird das Sharing angewendet (Schlüter 2018).

Viele Interviewprojekte wie das Projekt MenschenLeben an der Österreichischen Mediathek sind inzwischen online abrufbar. Die seit 1983 laufende Buchreihe „Damit es nicht verloren geht ..." veröffentlichte dutzende Autobiografien. Ich erinnere mich an eine 1995 vom Österreichischen Gedenkdienst organisierte Konferenz über das Exil von Flüchtlingen aus Nazi-Deutschland in Shanghai, zu der viele Zeitzeugen und Zeitzeuginnen aus aller Welt kamen. Eine Tochter aus einer Shanghai-Familie stand auf, so meine Erinnerung, und bedankte sich schluchzend für das Teilen und Mitteilen all ihrer Geschichten, das helfe ihr sehr. Solche Momente sind einer von vielen Gründen, warum wir Oral Historians den Menschen, die uns ihre Lebensgeschichten erzählen, in Dankbarkeit verbunden sind, denn sie helfen nicht nur uns bei der Forschung, sondern auch anderen. Gerda Lerner, eine US-amerikanische, feministische Historikerin österreichisch-jüdischer Herkunft schrieb:

The past becomes part of our present and thereby part of our future. We act individually and collectively in a process over time which builds the human enterprise and tries to give it meaning. Being human means thinking and feeling; it means reflecting on the past and visioning into the future. We experience; we give voice to that experience; others reflect on it and give it new form. That new form, in its turn, influences and shapes the way next generations experience their lives. That is why history matters. (Lerner 1997, S. 211)

An diesem Projekt für die Zukunft, können wir fächerübergreifend, verantwortungsvoll und reflektiert zusammenarbeiten, denn wie Gerda Lerner schrieb: „All human beings are practicing historians. As we go through life we present ourselves to others through our life story ..." (ebd., S. 199).

Danksagung Vielen Dank an Michael John, Elisabeth Pozzi-Thanner, Hildegard Pruckner, Dorit B. Whiteman und Jessica Wiederhorn.

Funding Open access funding provided by Paris Lodron University of Salzburg. 
Open Access Dieser Artikel wird unter der Creative Commons Namensnennung 4.0 International Lizenz veröffentlicht, welche die Nutzung, Vervielfältigung, Bearbeitung, Verbreitung und Wiedergabe in jeglichem Medium und Format erlaubt, sofern Sie den/die ursprünglichen Autor(en) und die Quelle ordnungsgemäß nennen, einen Link zur Creative Commons Lizenz beifügen und angeben, ob Änderungen vorgenommen wurden.

Die in diesem Artikel enthaltenen Bilder und sonstiges Drittmaterial unterliegen ebenfalls der genannten Creative Commons Lizenz, sofern sich aus der Abbildungslegende nichts anderes ergibt. Sofern das betreffende Material nicht unter der genannten Creative Commons Lizenz steht und die betreffende Handlung nicht nach gesetzlichen Vorschriften erlaubt ist, ist für die oben aufgeführten Weiterverwendungen des Materials die Einwilligung des jeweiligen Rechteinhabers einzuholen.

Weitere Details zur Lizenz entnehmen Sie bitte der Lizenzinformation auf http://creativecommons.org/ licenses/by/4.0/deed.de.

\section{Literatur}

Apel, L. (2018). Erinnern, erzählen, deuten. Oral History in der universitären Lehre. BIOS - Zeitschrift für Biographieforschung, Oral History und Lebensverlaufsanalysen, 1, 23-34.

Bajohr, F., \& Löw, A. (Hrsg.). (2016). The holocaust and the European societies: social processes and social dynamics. London: Palgrave Macmillan.

Bar-On, D. (1996). Die Last des Schweigens. Gespräche mit Kindern von Nazi-Tätern. Reinbek: Rowohlt. Berkowitz, H. (1998). Aber kommen Sie nicht zurück, Herr Berkowitz! In D. Ellmauer, A. Lichtblau \& H. Embacher (Hrsg.), Geduldet, geschmäht und vertrieben. Salzburger Juden erzählen (S. 158-168). Salzburg, Wien: Otto Müller.

Diekmann, I., \& Schoeps, J.H. (Hrsg.). (2002). Das Wilkomirski-Syndrom. Eingebildete Erinnerungen oder von der Sehnsucht, Opfer zu sein. Zürich, München: Pendo.

Epstein, H. (1979). Children of the holocaust: conversations with sons and daughters of survivors. New York: Puntman.

Ghitis, S. (2004). Reflecting on the unexpected. In Oral History Association Conference, Portland. Paper.

Halbmayr, B. (2015). Chancen und Probleme der Sekundäranalyse von ZeitzeugInneninterviews in der historischen Forschung. Westfälische Forschungen. Zeitschrift des LWL-Instituts für westfälische Regionalgeschichte, 65, 293-305.

Helige, B., John, M., Schmucker, H., Wörgötter, G., \& Wisinger, M. (2013). Endbericht der Kommission Wilhelminenberg. Wien: Domus. http://www.kommission-wilhelminenberg.at/presse/jun2013/ Bericht-Wilhelminenberg-web_code.pdf. Zugegriffen: 7. Dez. 2020.

Hirsch, M. (2012). The generation of Postmemory: writing and visual culture after the holocaust. New York: Columbia University Press.

John, M., \& Lichtblau, A. (1990). Schmelztiegel Wien - einst und jetzt. Zur Geschichte und Gegenwart von Zuwanderung und Minderheiten. Wien, Köln: Böhlau.

Kellermann, N. F. P. (2009). Holocaust trauma: psychological effects and treatment. New York, Bloomington: iUniverse.

Lerner, G. (1997). Why history matters: life and thought. New York, Oxford: Oxford University Press.

Lichtblau, A. (2002). Keeping distance. In P. Denis \& J. Worthington (Hrsg.), The power of oral history. Memory, healing and development. XIIth International Oral History Conference, Pietermaritzburg, South Africa (S. 814-824). Pietermaritzburg: Selbstverlag.

Lichtblau, A. (2006). Erinnerungsbilanz/Recapitulation of Memory, Dokumentarfilm 35 min. Salzburg: Selbstverlag.

Lichtblau, A. (2018). Oral History lässt sich nicht unterrichten? BIOS - Zeitschrift für Biographieforschung, Oral History und Lebensverlaufsanalysen, 1, 9-22.

Lichtblau, A. (2022). „Scham“ in Interviews mit Überlebenden des KZs Mauthausen. In A. Prenninger, G. Botz \& R. Fritz (Hrsg.), Leben und Erinnern nach Mauthausen. Europa in Mauthausen, Bd. 4. Wien, Köln, Weimar: Böhlau.

McCarthy, E. (2010). "Is oral history good for you?" taking oral history beyond documentation and into a clinical setting: first steps. The Oral History Review, 37(2), 159-169. https://doi.org/10.1093/ohr/ ohq094.

Pruckner, H. (2021). Psychodrama mit traumatisierten Menschen. Trauma. Zeitschrift für Psychodrama und Soziometrie, 20(1). 
Reiter, M. (2006). Die Generation danach. Der Nationalsozialismus im Familiengedächtnis. Innsbruck, Wien, Bozen: StudienVerlag.

Reiter, M. (2010). Vaterbilder und Mutterbilder. Geschlechtsspezifische Zuschreibungen von Täterschaft und Schuld in der NS-Nachfolgegeneration. In M. Figge, K. Hanitzsch \& N. Teuber (Hrsg.), Scham und Schuld. Geschlechter(sub)texte der Shoah (S. 61-79). Bielefeld: transcript.

Rickard, W. (1998). "More dangerous than therapy"?: interviewees' reflections on recording traumatic or taboo issues. Oral History (Memory, Trauma and Ethics), 26(2), 34-48.

Robins, R. S., \& Post, J. M. (1997). Political paranioa: the Psycholopolitics of hatred. New Haven, London: Yale University Press.

Rosenthal, G. (Hrsg.). (1997). Der Holocaust im Leben von drei Generationen. Familien von Überlebenden der Shoah und von Nazi-Tätern. Gießen: Psychosozial-Verlag.

Schlüter, C. (2018). „Ich teile mit dir ...“. Z Psychodrama Soziom, 17, 203-213. https://doi.org/10.1007/ s11620-018-0446-9.

Spiegelman, A. (2011). MetaMaus: a look inside a modern classic, Maus. New York: Pantheon Books.

Staub, E. (1989). The roots of evil: the origins of genocide and other group violence. New York: Cambridge University Press.

Staub, E. (2015). The roots of goodness \& resistance to evil. New York: Oxford University Press.

Vickers, E. L. (2019). Unexpected trauma in oral interviewing. The Oral History Review, 46(1), $134-141$. https://doi.org/10.1093/ohr/ohy099.

von Plato, A. (2004). Geschichte und Psychologie - Oral History und Psychoanalyse. Problemaufriss und Literaturüberblick. FQS. Forum Qualitative Sozialforschung, 5/1/18. https://www.qualitativeresearch.net/index.php/fqs/article/view/656. Zugegriffen: 5. Dez. 2020.

Wardi, D. (1992). Memorial candles: children of the holocaust. London: Routledge.

Welzer, H. (2009). Die Nachhaltigkeit historischer Erfahrungen. Eine sozialpsychologische Perspektive. In H. Radebold, W. Bohleber \& J. Zinnecker (Hrsg.), Transgenerationale Weitergabe kriegsbelasteter Kindheiten. Interdisziplinäre Studien zur Nachhaltigkeit historischer Erfahrungen über vier Generationen (S. 75-93). Weinheim, München: Juventa.

Whiteman, D. B. (1995). Die Entwurzelten. Jüdische Lebensgeschichten nach der Flucht 1933 bis heute. Wien, Köln, Weimar: Böhlau.

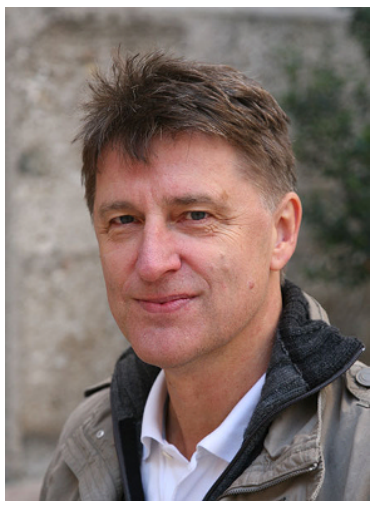

Univ. Prof. em., Dr. Albert Lichtblau Jahrgang 1954, Univ. Prof. em., Dr., Studium der Geschichte und Politikwissenschaft an der Universität Wien. Bis 2019 Universitätsprofessor am Fachbereich Geschichte und stellvertretender Leiter des Zentrums für jüdische Kulturgeschichte der Universität Salzburg. Forschungsschwerpunkte und Publikationen: Zeitgeschichte, Oral History, Audiovisuelle Geschichte, Migration, Nationalsozialismus, Genozidforschung, Rassismus, Erinnerungspolitik. 